\title{
Los desafíos de la agenda transnacional subestatal: la producción de nuevas energías en la provincia de Jujuy (Argentina)
}

\author{
The challenges of the transnational sub-state agenda. The \\ production of new energies in the province of Jujuy (Argentina)
}

Stella Justea

${ }^{a}$ Universidad Católica de Santiago del Estero, Argentina. stellajuste@gmail.com

\section{RESUMEN}

El presente artículo propone el estudio de la gestión transnacional de unidades subestatales y el desarrollo de nuevas. El caso de análisis es la gestión transnacional de provincia de Jujuy (Argentina) en referencia a la producción de carbonato de litio y energía solar, entre los años 2010 y 2017. El recorte temporal parte del primer proyecto de producción de carbonato de litio y finaliza con la construcción del parque solar de Cauchari en 2017.

El supuesto de análisis indica que las unidades subestatales emplean la gestión transnacional como estrategia para la persecución de sus objetivos de desarrollo, a través de proyectos energéticos que trabajan en articulación con el Estado y otros actores internacionales.

El objetivo es describir las estrategias de Jujuy, respecto del Estado y del sistema internacional, para la gestión de proyectos energéticos que posibiliten el desarrollo local.

A tal fin se emplean las teorías de la interdependencia compleja que avalan la existencia de un sistema internacional compuesto por actores de distinta naturaleza, y los estudios de la paradiplomacia para adentrarse en la acción internacional de las unidades subestatales y su vinculación con el desarrollo local.

La metodología es cualitativa, empleando entrevistas, análisis de fuentes bibliográficas y documentales.

Palabras clave: Estado; unidad subestatal; gestión transnacional; desarrollo; energía

\section{ABSTRACT}

This article proposes to study the transnational management of sub-state units on energy production. The case of analysis is the province of Jujuy (Argentina) in reference to the production of lithium carbonate and solar energy, between 2010 and 2017. The period under study begins with the first project of lithium carbonate production and it ends with the solar plant construction of Cauchari in 2017.

The hypothesis of analysis indicates that sub-state units use transnational management as a strategy in order to pursue its development goals through energy projects with the State and other international actors.

The objective is to describe Jujuy's strategies, regarding the State and the international system, for the management of energy projects that enable local development.

To this end, this article uses the theories of complex interdependence that support the existence of an international system composed of actors of different natures, and paradiplomacy studies to understand the international action of sub-state units and their link with local development.

Recibido: 15 de marzo de 2018. Aceptado: 29 de junio de 2020. 
The methodology is qualitative, using interviews, analysis of bibliographic and documentary sources.

Keywords: State; sub-state unit; transnational management; development; energy

\section{INTRODUCCIÓN}

En el campo de las Relaciones Internacionales, la gestión internacional de las unidades subestatales despertó interés en la década de 1970, en el marco de las transformaciones que modificaron el carácter estadocéntrico del sistema internacional (Keohane y Nye, 1989; Rosenau, 1997; Del Arenal, 2009). Este fenómeno propició la aparición de nuevos actores, gubernamentales y no gubernamentales, que conformaron un entramado de relaciones intergubernamentales y transnacionales (Keohane y Nye, 1989, pp. 7-8). Este enfoque, conocido como "interdependencia compleja", es una crítica a la corriente realista y se sustenta en un contexto global de transformación que propicia la aparición de actores de distinta naturaleza (Keohane y Nye, 1989, p. 162; García Segura, 2014, p. 302). Sin embargo, la gestión internacional de las unidades subestatales no significó el declive o la sustitución de la política exterior de los Estados, sino más bien, puso en evidencia la complejidad del sistema internacional, donde cada actor irrumpe con sus propios intereses (Zeraoui, 2009, p. 17).

Como unidades políticas que integran un Estado, la actuación inmediata de las unidades subestatales es su propio territorio, el cual administra y sobre el cual se han producido procesos socioeconómicos y políticos de carácter sistémico que impactan en ellas (Calvento, 2016, p. 299).

En este sentido, las acciones de la provincia de Jujuy son abordadas desde el concepto de gestión transnacional subestatal, entendiendo que la misma comprende acciones internacionales que ocurren en dos subsistemas: estatal e internacional. Entonces, se entiende la gestión transnacional subestatal como aquella política pública desplegada por unidades subestatales en el sistema estatal y en el sistema internacional, y que tienen como fin responder a los intereses de desarrollo subestatal (Juste, 2017, pp. 180-181).

La actuación de las provincias como actores internacionales no es nueva. Basta recordar la historia de las ciudades italianas, los landers alemanes, los cantones suizos, así como las provincias argentinas en la formación del Estado nacional. En las décadas recientes, los cambios en el sistema internacional dieron impulso al accionar de provincias, regiones, departamentos y municipios en el plano internacional que, a su vez, crearon una novel comunidad epistémica, conocida como paradiplomacia o internacionalización territorial.

El término paradiplomacia fue acuñado por el profesor Soldatos en la década de 1980, pero el canadiense Duchacek fue quien lo desarrolló, convirtiéndose en uno de sus máximos exponentes. Duchacek (1990, p. 15) entendió la paradaplomacia como "las entradas directas e indirectas de los gobiernos no-centrales al campo de las relaciones internacionales", poniendo énfasis en el prefijo "para" porque entendió que esta capacidad de los gobiernos locales era "paralela", es decir "subsidiaria o accesoria" respecto a la política exterior ${ }^{1}$ de los Estados (Duchacek,

\footnotetext{
${ }^{1}$ Siguiendo la línea de pensamiento clásico, Sarquís sostiene que la política exterior fluye "de adentro hacia afuera”, a diferencia de la política internacional que circula en el sentido opuesto.
} 
1990, p. 25). Sin embargo, la definición más clara del concepto, la aportó más tarde el profesor Noé Cornago:

La paradiplomacia puede ser definida como la participación de los gobiernos no centrales en las relaciones internacionales, a través del establecimiento de contactos permanentes o ad hoc con entidades públicas o privadas extranjeras, con el propósito de promover diversos aspectos socioeconómicos o culturales, así como cualquier otra dimensión exterior de sus propias competencias constitucionales (Cornago, 2001, p. 2).

Particularmente, Argentina receptó estas nuevas dinámicas, reconociendo la capacidad de actuación internacional de sus provincias a través de la reforma constitucional del año $1994^{2}$.

En el contexto nacional, la producción energética se visualiza como una nueva oportunidad de reinserción de Argentina en el mundo. Este sector ha posibilitado que durante los últimos años se multiplicaran las señales de inversión para el desarrollo de proyectos en las provincias de Jujuy, Salta y Catamarca, ubicadas en la periferia norte del Estado. Teniendo en cuenta lo anterior, el presupuesto de partida del presente artículo sostiene que la gestión transnacional de Jujuy en materia energética se vislumbra como una herramienta de desarrollo local que articula actores de diversa naturaleza, y busca reposicionar los intereses locales en la agenda del Estado ${ }^{3}$.

Como actor subestatal, se entiende que la provincia despliega su accionar en dos planos o subsistemas que conforman la gestión transnacional. Por un lado, realizan acciones frente al Estado el cual integran (sistema estatal), a través de una gestión estatal. Y, por otro lado, despliegan un accionar fuera de las fronteras estatales con actores internacionales (sistema internacional), llevando a cabo gestiones internacionales. El accionar en ambos planos conforma la gestión transnacional (Juste, 2017).

Entonces, la política exterior es una respuesta a las influencias y estímulos procedentes del contexto externo que se retroalimenta de forma constante. La política internacional en cambio, no está diseñada por un actor en particular, no constituye un plan de acción propiamente dicho, sino que surge como resultado de la manera en que se diseñan y se implementan las políticas exteriores estatales en el sistema internacional (Sarquís, 2009, pp, 37-38).

2 En su artículo 124, la Constitución Nacional (1994) dejó establecido que las provincias: (...) las provincias podrán también celebrar convenios internacionales en tanto no sean incompatibles con la política exterior de la Nación y no afecten las facultades delegadas al Gobierno federal o el crédito público de la Nación; con conocimiento del Congreso Nacional (Art.124).

3 Prebisch en América Latina y posteriormente Wallerstein en Europa, identificaron un sistema internacional jerarquizado en zonas centro y zonas periféricas. Para Wallerstein los países de las regiones periféricas son economías agrícolas o mineras, productoras de materias primas que carecen de poder. Por el contrario, los países del centro, son economías industrializadas que detentan una posición dominante en el sostenimiento de las estructuras políticas (Wallerstein, 1979: 490 y ss.). los referentes de la CEPAL, entre ellos Raúl Prebisch, entendieron que la dinámica del sistema internacional era definida por un patrón de desarrollo dominante que establece condiciones de desarrollo en el centro, y subdesarrollo en la periferia (Prebisch, 2008, pp. 28-29). 
Tabla 1: Planos de acción de la gestión transnacional subestatal.

\begin{tabular}{|l|}
\hline Gestión Transnacional Subestatal \\
\hline Sistema Estatal (gestión estatal) \\
\hline Sistema Internacional (gestión internacional) \\
\hline
\end{tabular}

Fuente: elaboración propia.

La provincia de Jujuy, ubicada en el extremo norte de Argentina, posee una larga tradición de explotación de sus riquezas mineras que, junto a la producción azucarera y tabacalera, son las actividades económicas emblemáticas de la región (MECON, 2016, p. 3-4). No obstante, a partir de los descubrimientos geológicos acaecidos en el año 2008, Jujuy se encaminó hacia la exploración y explotación de nuevos recursos energéticos, articulando actores locales e internacionales.

A tales efectos, se analizarán los proyectos energéticos anclados en la provincia, y el protagonismo de Jujuy respecto del Estado y otros actores internacionales intervinientes. La metodología propone un análisis esencialmente cualitativo a partir de entrevistas a actores gubernamentales y del sector privado que intervienen en ambos casos de estudio, junto al análisis de fuentes bibliográficas y documentales.

El trabajo se estructura en cuatro partes. La primera de ellas aborda el rol de la provincia en la producción de nuevas energías. Seguidamente se aborda la conformación del "triángulo del litio" en Sudamérica. Luego, una tercera parte analiza la gestión y la potencialidad de la energía solar en la puna jujeña. Y, por último, algunas reflexiones finales.

\section{EL ROL DE LA PROVINCIA EN LA PRODUCCIÓN ENERGÉTICA}

El interés actual de inversores extranjeros en los recursos energéticos de Argentina se complementa no solo con el contexto internacional y regional, sino también, con las políticas de promoción impulsadas por las provincias del norte del país, económicamente rezagadas. En el caso de Jujuy, la provincia elaboró un paquete de políticas que incluyeron beneficios de exención impositiva en los primeros años y creó una empresa estatal para la conformación de sociedades con empresas interesadas en los recursos locales (Castello y Kloster, 2015, p. 62).

El gobierno nacional propició en 2012 la creación de la Organización Federal de los Estados Mineros (OFEMI), conformada por las provincias mineras no metalíferas (Salta, Jujuy, Catamarca, San Juan, Mendoza, Neuquén, Río Negro, Chubut y Santa Cruz.). La OFEMI se organizó con el fin promocionar acciones para el desarrollo minero nacional e incentivar mecanismos para la incorporación de valor agregado industrial a los recursos minerales primarios. Este organismo federal es actualmente presidido por la provincia de Jujuy.

A nivel nacional, Jujuy es la provincia que más ha avanzado en el desarrollo de políticas públicas locales relacionadas estrictamente a proyectos mineros. Una de las estrategias empleadas por la provincia para maximizar los beneficios de la explotación minera y energética, fue la creación de la empresa estatal JEMSE "Jujuy Energía y Minería Sociedad del Estado”, a través del decreto $\mathrm{N}^{\circ} 7626 / 11$, ratificado por la ley $\mathrm{N}^{\circ} 5676$ en el año 2011. El objetivo del gobierno local fue crear una unidad 
de negocios del Estado provincial con capacidad de negociación, asociación y comercialización que el Estado provincial en su calidad de tal, no posee.

De esta forma, JEMSE se creó con amplias atribuciones, entre las cuales se encuentran:

- Presentarse a licitación pública o privada a nivel provincial, nacional o internacional en forma individual o asociada.

- Solicitar permisos y concesiones mineras, exenciones y otras medidas impositivas o de promoción.

- Establecer áreas de exploración geológica minera en los términos del artículo 346 del Código de Minería4.

- Desarrollar obras de infraestructura, la prestación de servicios, el otorgamiento de préstamos y subsidios, la capacitación de personal, la prestación de asistencia técnica, el abastecimiento de equipos e insumos, la compra/venta de productos mineros. A tal fin, se la faculta para celebrar toda clase de actos jurídicos en el país o en el extranjero con personas físicas o jurídicas5.

- Comercializar sustancias minerales.

- Investigar, explorar y/o explotar recursos mineros en la provincia por sí misma o por intermedio de terceros.

A través de JEMSE, Jujuy participa de las exploraciones y explotaciones referidas a hidrocarburos, minería, y energía, además del comercio industrial que, en principio, a todo Estado provincial le está vedado por ley. Esta participación otorga a la provincia una forma de intervención alternativa, funcionando JEMSE como una unidad de negocios que permite al Estado provincial adquirir control sobre la producción minera, los proyectos energéticos y, además, redunda en beneficios para los inversores que se asocien a ella.

Otra de las estrategias implementadas por la provincia fue la declaración del litio como "mineral estratégico para el desarrollo", a través del decreto provincial 7592/11. Este instrumento supuso, entre otras medidas, la creación de la "Unidad de Gestión Ambiental": un comité de expertos integrados por especialistas ministeriales, representantes de empresas, asesores externos, representantes de las comunidades que habitan la zona de la explotación, entre otros. El objeto principal de la Unidad de Gestión Ambiental es la evaluación de impacto ambiental en proyectos que involucran la exploración y explotación del mineral, actuando como un órgano que autoriza la ejecución de estos, pudiendo sugerir cambios o negar la aprobación de su ejecución.

Al respecto de las estrategias para alcanzar el desarrollo, las teorías

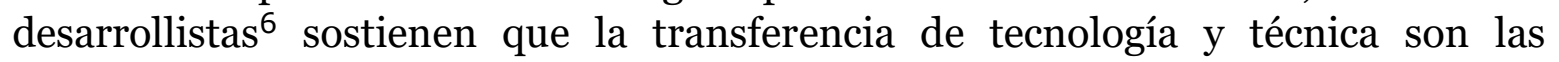
herramientas que deben trabajar las economías periféricas, como el caso de Jujuy,

4 El Código de Minería de Argentina establece en su artículo No 346 que: "La investigación geológico-minera de base que realice el Estado Nacional en todo el país y las que efectúen las provincias en sus territorios es libre y no requiere permiso de la autoridad minera".

5 Decreto provincial $\mathrm{N}^{\circ} 7626$, año 2010.

${ }^{6}$ Los teóricos desarrollistas (Cardoso y Faletto, 1977; Prebisch; 2008; Dos Santos, 2011) coincidieron en defender la relevancia de la transferencia de tecnología y técnicas como una estrategia para reducir la dependencia a los centros económicos del sistema. 
para superar su dependencia de los centros económicos. Todo ello conlleva a pensar que la producción propia de conocimiento científico, tecnológico y la innovación que se traduzcan en herramientas de desarrollo, exigen un sistema de coordinación entre el gobierno provincial, las instituciones científicas y la estructura productiva, que deben funcionar de manera articulada (Prebisch, 2008, pp. 34-39). Bajo estos parámetros, las inversiones del noreste asiático posibilitarían a provincias periféricas como Jujuy, la transferencia de tecnologías y técnicas adecuadas a innovar los procesos productivos en el área energética, sin depender de las transferencias predeterminadas por la región central argentina. Junto a políticas públicas (locales y nacionales) destinadas a la generación de conocimiento y con los aportes de la inversión extranjera, las economías periféricas pueden propender al desarrollo de técnicas y tecnologías propias.

En el contexto nacional y provincial, existe un marcado consenso sobre la necesidad de industrializar la producción minera. Por tal motivo, Jujuy buscó estrategias que vinculen la extracción de salmueras de litio con un proceso de industrialización. Siguiendo esta línea, se conformó el Consorcio Asociativo PúblicoPrivado (CAPP) "Litio Argentino". El mismo se constituyó a inicios del año 2016 entre la Universidad Nacional de Jujuy (a través del Centro de Investigación y Transferencia - CIT) y las empresas Laring San Luis S.A. e YPF Tecnología S.A (Fornillo, 2009, pp. 74-79). El consorcio tiene como objetivo la creación de una plataforma de investigación y desarrollo de tecnologías para la extracción del litio y su procesamiento a fin de producir compuestos aplicables en baterías.

Los actores involucrados en el proyecto se asociaron en base a la convocatoria del Fondo Sectorial Argentino (FONARSEC) que luego se traduciría en la instalación de un Centro de Referencia de Litio en Jujuy y la formulación del proyecto "Litio Argentino", con líneas de investigación financiadas por FORNASEC. No obstante, y si bien son notables los esfuerzos del gobierno local por contribuir a la modernización del entramado regional económico, no se vislumbran aún resultados concretos sobre el agregado de valor en los productos mineros. Por el momento la coordinación entre las empresas, el gobierno provincial y la Universidad, se encuentra en una fase de planificación, investigación y articulación. Muestra de esto, es el hecho de que Jujuy ha sido sede de las recientes reuniones de la OFEMI, la reunión de los rectores de la Universidades Nacionales y sede del Tercer Congreso Internacional de Litio.

En esta dinámica, el cambio de liderazgo político en el Estado y en la provincia no ha introducido cambios sustanciales respecto a la actividad referida al litio, manteniendo la idea de la industrialización del mineral como una de las políticas prioritarias. Así, el gobernador Gerardo Morales inaugura el Centro de Desarrollo Tecnológico "Gral Manuel Savio", más conocido como el "Instituto del Litio", en agosto de 2017. Esta institución se compone de tres áreas de desarrollo científico- tecnológico:

- El Instituto de Datación y Arqueometría de Jujuy (INDyA)

- El Centro de Investigación y Desarrollo en Materiales Avanzados y Almacenamiento de Energía de Jujuy (CIDMEJu)

- El Instituto Jujeño de Energías Renovables y Eficiencia Energética.

Además de estos avances, la provincia firmó en el mismo momento, un acuerdo con la empresa italiana Seri Group para la fábrica de baterías y celdas de 
litio y fábrica de material activo. A través de JEMSE, la provincia receptó 2 millones y medio de dólares para el ensamble de baterías que se prevé para finales de 2018, además de 49 millones de dólares para la construcción de un módulo que produzca celdas de litio. Por último, el acuerdo prevé la construcción de producción de material activo, que se prevé para el año 2022 y que requerirá una nueva inversión (El Tribuno de Jujuy, 2017).

\section{LA CONFORMACIÓN DEL TRIÁNGULO DEL LITIO SUDAMERICANO}

América Latina ha sido históricamente reconocida por la riqueza de los recursos que posee gracias a sus condiciones naturales geológicas y medioambientales. Específicamente, Argentina, Bolivia y Chile concentran alrededor del $60 \%$ de las reservas de litio, conformando la zona denominada “Triángulo del Litio" o "Triángulo ABC”. En esta zona, Bolivia concentra en el Salar de Uyuni el 27,3\% de los recursos identificados de litio, Chile representa el 22,7\% a través del Salar de Atacama. En Argentina, las reservas de mayor relevancia se encuentran en los salares de Olaroz y Cauchari (en Jujuy), los salares de Rincón, Pocitos y Arizaro (Salta), y el salar del Hombre Muerto (Catamarca), que juntos suman alrededor del 8\%7 (Comisión Nacional de Litio de Chile, 2015, pp. 11-13).

En el panorama regional, Jujuy ha resultado atractiva a las inversiones debido a las circunstancias restrictivas para la explotación del mineral en Bolivia y Chile. Las particularidades presentes en cada Estado, propiciaron que las miradas de los inversores se voltearan hacia Argentina que presentaba mayores ventajas comparativas y un marco jurídico de mayor flexibilidad respecto a sus pares fronterizos. Así surgieron en Jujuy dos ambiciosos proyectos impulsados por inversiones japonesas, australianas y coreanas, que se asociaron a la empresa pública provincial Energía y Minería Sociedad del Estado (JEMSE).

A continuación, se desarrollarán las características de cada proyecto y luego, el rol del gobierno provincial respecto del mismo.

\subsection{El Proyecto Olaroz}

El Salar de Olaroz se ubica en la zona de puna o desierto de altura, a 4.540 metros sobre el nivel del mar, en el departamento de Susques. El proyecto se inició en 2009 a través de una inversión de 330 millones de dólares estadounidenses. Esta inversión inicial se gestionó mediante un grupo inversor que conformó un joint venture del cual participaron los siguientes actores 8 :

- La automotriz japonesa Toyota, a través de su empresa abastecedora Toyota Tsusho Corp, con el 25\% del capital.

- La empresa minera australiana Orocobre, que opera en Jujuy a través de la empresa Sales de Jujuy S.A., participó con el 66,5\%.

7 El Salar de Atacama (Chile) es el segundo depósito de litio más grande del conglomerado, siendo superado por el Salar de Uyuni en Bolivia. Sin embargo, Chile presenta una notable ventaja económica por ser el de mayor concentración y calidad y por reunir las mejores condiciones climáticas para la evaporación, disminuyendo el costo de procesamiento (Comisión Nacional de Litio de Chile, 2015, pp. 11-13).

8 Datos proporcionados por la Secretaría de Minería e Hidrocarburos, perteneciente al Ministerio de Producción del Gobierno de la Provincia de Jujuy, abril de 2016. 
- La empresa estatal de la provincia de Jujuy, JEMSE (Jujuy Energía y Minería Sociedad del Estado), participó con el 8,5\%.

En términos absolutos, la inversión proveniente de la empresa japonesa alcanzó los 82,5 millones de dólares, y la empresa australiana Orocobre se posicionó como la mayor inversora aportando casi 220 millones de dólares (Slipak, 2015, p. 97).

Con la intervención de estos tres actores, la planta de Olaroz comenzó a trabajar en el año $2015 \mathrm{y}$, al completar el primer año de funcionamiento, la producción alcanzó las 17.500 toneladas de litio y 20.000 toneladas de cloruro de potasio. Para comprender el nivel de productividad de la planta de Olaroz, estas cifras equivalen al consumo total de litio actual de economías como Japón, mientras que a nivel global representan alrededor del 10\% del consumo mundial.

En comparación a sus pares de la región, la principal ventaja que presenta el salar de Olaroz es la escasa presencia de magnesio, componente que se encuentra en la salmuera que se extrae. En Olaroz la relación con el magnesio es muy baja debido a las condiciones geológicas. Los especialistas consideran que es del 2,8\% y que este índice lo diferencia positivamente frente al salar de Atacama (Chile) donde el índice es de 6,4\%, y el salar de Uyuni (Bolivia) que es de 19\% (Slipak, 2015, p. 96).

De manera descriptiva y general, la obtención de carbonato de litio consiste en la extracción de salmuera a partir de perforaciones realizadas en el salar. La salmuera obtenida es trasladada a piletas de evaporación donde se concentra mediante el uso de radiación solar. Luego, se procesa a través de una planta que precipita el magnesio y lo traslada a otras piletas con el magnesio eliminado. Finalmente, la salmuera desprovista de magnesio se coloca en otras piletas de evaporación antes de procesarla en un circuito de la planta de carbonato de litio. El producto final de la salmuera de Olaroz se traduce en carbonato de litio con una concentración de más del 99,5\%, convirtiéndola en apta para la producción de baterías (Sales de Jujuy, 2017).

Con este panorama productivo, se produjo la primera exportación de carbonato de litio jujeño con destino a Japón en abril de 2015 (Télam, 2015). Y, en 2019, el gobierno provincial junto a la empresa, anunciaron ampliación de la producción a 42.500 toneladas para el año 2021, con una proyección aproximada a 300 millones de dólares (Dirección de Prensa del Gobierno de Jujuy, 2019).

\subsection{Proyecto Olaroz-Cauchari}

También el proyecto Olaroz-Cauchari se emplaza en la puna jujeña, en el departamento de Susques. La inversión inicial para el proyecto de producción de carbonato de litio se originó luego de un convenio de cooperación firmado entre la empresa Lithium Americas Corp. junto a su empresa subsidiaria EXAR y la empresa coreana Posco. El acuerdo entre estos actores propició el inicio de la construcción de una planta piloto en el año 2014 con una inversión inicial de 30 millones de dólares. Finalizada esta prueba piloto, Posco invirtió alrededor 260 millones de dólares más para la planta industrial final (La Política Online, 2015).

La construcción de la planta piloto formó parte de una primera etapa de experimentación y evaluación que tuvo como fin determinar la calidad del carbonato de litio. Si bien se trata de una planta piloto, la misma se diseñó con una capacidad 
productiva de 200 a 500 toneladas aproximadamente, que representan un nivel de producción industrial (La Política Online, 2015).

En comparación al proyecto Olaroz, la principal característica de esta iniciativa fue la implementación de una tecnología de vanguardia compatible con el cuidado del medioambiente, que aportó la empresa coreana Posco. El empleo de la tecnología coreana disminuyó la cantidad de pozos de evaporación de salmuera, reduciendo significativamente el impacto ambiental en el lugar.

A mediados de mayo del año 2016, la empresa SQM de Chile se integra al proyecto. De esta manera, la empresa EXAR queda conformada por un capital accionario dividido 50\% entre Lithium Americas Corp, de Canadá, y 50\% de la firma SQM de Chile. Para su ingreso al proyecto, EXAR aportó 500 millones de dólares, y está previsto que para el primer semestre del año 2019 la producción ronde alrededor de 25.000 toneladas de carbonato de litio grado batería por año (EconoJournal, 2016). A inicios del año 2020, fue presentada una actualización del proyecto, previéndose una producción de carbonato de litio de 40.000 toneladas por año a partir de 2021 (Panorama Minero, 2020).

\section{LA ENERGÍA SOLAR EN LA PUNA JUJEÑA}

A finales de año 2015, la preocupación por el desarrollo de nuevas fuentes energéticas de las grandes economías, tales como China, motivó a que otros países y sus unidades subestatales, que vieron allí una oportunidad de desarrollo en base a sus condiciones y recursos ambientales.

En particular, el interés chino estuvo estrechamente ligado a la necesidad de asegurar el suministro energético que garantice la continuidad de su crecimiento económico (Schujovitzky, 2017, p. 36). En esta línea, el Estado chino dispersó numerosas inversiones en proyectos de materia energética en distintos puntos geográficos.

En América Latina, donde los países demandan abundantes recursos en forma de inversión y de financiamiento, China encontró un campo abierto para explorar y consolidar su presencia en la región, mientras que los Estados latinoamericanos vieron esta presencia como una oportunidad de crecimiento. Este interés complementario facilitó la vinculación y el trabajo conjunto de distintos actores que se involucraron a fin de propiciar el proyecto de energía solar de Cauchari en la puna jujeña.

A nivel estatal, el gobierno de Mauricio Macri, iniciado a fin de 2015, reorientó la política exterior argentina respecto de la gestión anterior de Cristina Fernández, especialmente a través del regreso al mercado internacional de capitales y la normalización de los vínculos políticos con las principales economías del mundo occidental (Oviedo, 2017, p. 13).

A partir de la nueva administración de la coalición Cambiemos, encabezada por Mauricio Macri, comenzó a visibilizarse la gestión transnacional de las provincias argentinas detrás de los proyectos de inversión que antes eran encarados directamente por el Estado federal. Un ejemplo de ello, fue la inclusión de los gobernadores de provincias con proyectos de inversión en la misión de Estado a China en mayo de 2017. 
A nivel subestatal, Jujuy también afrontó un cambio de administración a finales del año 2015, que coincidió con el cambio de signo político a nivel nacional ${ }^{9}$. Este hecho coadyuvó a sincronizar los intereses entre ambos niveles de gobierno, estatal y subestatal, e implementar acciones políticas conjuntas orientadas en un mismo sentido.

En este contexto, la IED de China dirigida hacia América Latina parece tener una triple finalidad. Por un lado, promover el desarrollo energético en los Estados latinoamericanos que permita garantizar su crecimiento. Por otro lado, crear oportunidades de inversión externa para las empresas chinas en desarrollo. Y, por último, contribuir al desarrollo de la infraestructura necesaria para el tránsito de productos desde y hacia China.

Uno de los antecedentes más relevantes para la relación chino-argentina en la administración de Macri, fue la aprobación del "Plan Quinquenal Integrado China-Argentina para la Cooperación en Infraestructura (2017-2021)". Este instrumento, que salió a la luz poco antes de la visita oficial del presidente a China en mayo de 2017, incluyó un anexo con 16 proyectos de "cooperación de infraestructura prioritaria", trabajados junto a las provincias y que se detectaron como de interés mutuo (Forbes Argentina, 2017).

En este marco, Argentina estableció como prioridad fortalecer la infraestructura de conexión que profundice el transporte de bienes entre los países del MERCOSUR y vincule al bloque sudamericano con el mercado asiático. Durante el foro "Una franja y una ruta para la cooperación internacional", el presidente Macri manifestó el deseo de vincular el MERCOSUR con la Alianza del Pacífico a través de la IIRSA (Iniciativa para la Integración de la Infraestructura Regional Sudamericana), con el objeto de mejorar la conectividad de la región (Infobae, 2017). Por lo tanto, Las intenciones argentinas se dirigieron principalmente a la obtención de financiamiento externo que posibilite optimizar la conectividad en la región para mejorar las condiciones de inserción en los mercados internacionales.

Desde China, los acuerdos de inversión promovidos tienen una base muy clara. Se trata de préstamos para inversión a tasas bajas (principalmente para infraestructura) a cambio de que las obras tengan una política de preferencias con los proveedores chinos (Télam, 2017). Este ha sido el mecanismo por el que los capitales de origen chino han penetrado en toda América Latina y África en las últimas dos décadas.

La visita de Estado a China en 2017, cristalizó el trabajo que las provincias y el Estado venían realizando en forma conjunta de cara al vínculo con el gigante asiático. En líneas generales, los tres ejes de trabajo que se formalizaron en el encuentro giraron alrededor de la infraestructura energética, transporte y minería. En el caso de Jujuy, la construcción de un parque solar en la puna de Cauchari (Susques, Jujuy) fue el proyecto que más elaborado llegó al momento de realizarse la visita oficial.

${ }_{9}$ El proceso electoral que llevó a Mauricio Macri a la presidencia del Estado argentino, fue el mismo que condujo al jujeño Gerardo Morales a la gobernación de la Provincia de Jujuy. Ambos, Macri y Morales, representaron al partido político PRO, una coalición de fuerzas políticas opositoras al Frente Para la Victoria (FPV), encabezado por Cristina Fernández. 
Con este marco antecedente, se analizarán los aspectos más relevantes de la gestión transnacional de Jujuy, frente al Estado nacional y otros actores internacionales, que derivaron en el proyecto Cauchari.

\subsection{La gestión transnacional jujeña en la captación de inversiones chinas}

A mediados de 2016, el gobierno nacional presentó el programa RenovAR con el objeto de transformar la matriz energética Argentina y, a su vez, propender a la conservación del medioambiente. El programa consistió en el desarrollo de proyectos de generación de energía, a través de licitaciones públicas nacionales. En la primera etapa, RenovAR licitó la generación de $600 \mathrm{Mw}$ eólicos, $300 \mathrm{Mw}$ solares fotovoltaicos, $65 \mathrm{Mw}$ de plantas de biomasa, $20 \mathrm{Mw}$ de mini centrales hidroeléctricas y $15 \mathrm{Mw}$ de biogas.

Bajo la administración de Gerardo Morales, Jujuy rubricó contratos con firmas chinas de gran porte para la primera convocatoria del programa RenovAR, a la cual se presentó con 3 proyectos de producción de energía solar de 100 Mw cada uno. Las empresas que se asociaron al proyecto fueron Power China, Shangai Electric y Telsun, todas firmas de origen chino. Como resultado, Jujuy se adjudicó $300 \mathrm{Mw}$ de los $300 \mathrm{Mw}$ para energía fotovoltaica licitados en la primera etapa del programa (Dirección de Prensa del Gobierno de Jujuy, 2016).

La propuesta jujeña se distinguió del resto por las características geológicas particulares del territorio jujeño, que la hacen sumamente competitiva. Tal apreciación se evidenció durante la definición del proceso licitatorio, en donde la propuesta de Jujuy se impuso sobre otros 59 proyectos del mismo rubro que provenían de distintos puntos del país. En líneas generales, el proyecto Cauchari se compone de tres parques fotovoltaicos (Cauchari 1, 2 y 3), diseñados para alcanzar una capacidad de generación de $100 \mathrm{Mw}$ cada uno, con un monto de inversión estimado de 340 millones de dólares.

Una vez adjudicados los $300 \mathrm{Mw}$ a la provincia, el proyecto se sumó a la agenda del Estado nacional respecto de la relación bilateral con China. Esto se debió a que, para la concreción de los parques, Jujuy requería la inversión de las firmas chinas que trabajaron el proyecto previo y, en este sentido, obtuvo el apoyo del Estado para impulsar las negociaciones.

Durante la visita oficial del presidente Macri a China en mayo de 2017, se incorporaron a la comitiva los gobernadores de las provincias con proyectos que requerían la captación de recursos externos para su ejecución. Entre esos proyectos que ya contaban con algún grado de avance en las negociaciones se encontraban: la rehabilitación de los sistemas ferroviarios de línea (San Martín, Roca y Mitre) y de carga (Belgrano y Urquiza), las centrales nucleares IV y V, la planta hidroeléctrica "El Tambolar" y las represas Kirchner y Cepernic, entre otros. En sostén a los proyectos que presentaban mayor grado de avance, la comitiva que acompañó al presidente, incluyó a los gobernadores de San Juan, Río Negro, y al gobernador de Jujuy, Gerardo Morales ${ }^{10}$. De todos los proyectos gestionados durante la visita,

\footnotetext{
${ }^{10}$ La provincia de San Juan esperaba la aprobación final para la construcción de la represa hidroeléctrica "El Tambolar", mientras que la intención de la provincia de Río Negro era concretar la firma de un convenio para el financiamiento de una quinta central nuclear.
} 
Cauchari fue el que captó la mayor atención, obteniéndose resultados concretos del viaje y evidenciándose una clara sincronía entre los intereses de ambos niveles de gobierno, estatal y subestatal.

Con el apoyo del Estado nacional, Jujuy acordó a través de JEMSE, las condiciones del vínculo societario con las firmas chinas Power China, Shanghai Electric y Talesun. El resultado fue la participación estatal provincial del 80\%, y el $20 \%$ restante a cargo de capitales privados distribuidos entre las firmas chinas. (Dirección de Prensa del Gobierno de Jujuy, 2016).

\subsection{Las ventajas comparativas de Cauchari}

En virtud de poseer condiciones geológicas y medioambientales favorables, la provincia logró aunar esfuerzos para el diseño del segundo parque solar más grande del mundo, con una potencia de 1.000 megavatios $(\mathrm{Mw})$, similar al parque solar existente en Dubai (Télam, 2017). El proyecto fue elaborado por la empresa Power China, a través de la empresa subsidiaria Shangai Electric, y presentado en el año 2017 al gobierno jujeño.

El parque contempla el funcionamiento de más de 1,2 millón de paneles solares, de acuerdo con el proyecto que presentó Power China y Shanghai Electric, mientras que la firma del mismo origen Talensun que tiene a su cargo la fabricación de los equipos que se instalarán en Cauchari (Télam, 2017).

La construcción de la planta dio inicio en octubre de 2017 en la localidad de Cauchari, departamento de Susques, a 4.000 metros de altura sobre el nivel del mar, generando 600 puestos de trabajo directo y cerca de 1000 indirectos (El Tribuno de Jujuy, 2017). Casualmente, Cauchari es la misma localidad en la que se emplaza el proyecto de producción de litio de la empresa EXAR. De esta manera, Cauchari concentra los tres parques solares más un proyecto de producción de litio ${ }^{11}$. Y, en líneas generales, el departamento de Susques en la puna jujeña, concentra la totalidad de los proyectos energéticos situados en la provincia.

A través de Cauchari 1, 2 y 3, Jujuy aspira a generar la mitad de su demanda eléctrica con energías renovables para 2019 y a aumentar su generación de energía solar 2,5 veces la demanda energética de la provincia en diez años (El Tribuno de Jujuy, 2017).

\subsection{El bono verde provincial}

Para la ejecución del proyecto Cauchari, quedó establecido que la participación de la provincia en la inversión inicial fuese del 80\% del proyecto. Con este panorama, el gobierno provincial se enfocó en gestionar los medios para cumplir con el financiamiento pactado. Así, en septiembre de 2017, un año después de haber ganado la licitación para producir los 300 Mw de energía solar, Jujuy colocó por primera vez un bono verde certificado por calificadora internacional de Argentina.

Los bonos verdes constituyen títulos de crédito emitidos por instituciones públicas o privadas, por un plazo fijo y, generalmente, a una tasa de interés fija, con el fin de incrementar el capital (Ecopost, 2016). Lo distintivo de los bonos verdes, es el compromiso por parte del emisor, en este caso Jujuy, de canalizar los fondos a

\footnotetext{
${ }^{11}$ El otro proyecto de extracción de salmuera de litio se sitúa en el Salara de Olaroz, también en el departamento de Susques (Jujuy).
} 
proyectos que tengan que o tengan impacto negativo en el medioambiente y sean sostenibles, es decir, que produzcan un efecto positivo en relación a la problemática del cambio climático (Ecopost, 2016). Para el caso de Jujuy, el objetivo de la emisión de deuda fue obtener parte del financiamiento que la provincia debía aportar al proyecto para iniciar la construcción de las plantas Cauchari 1, 2 y 3.

De esta forma, se concretó la primera emisión de deuda en dólares, a través de la colocación de un bono por 210 millones de dólares, a 5 años de plazo y a una tasa del 8,625\% anual. El bono fue emitido bajo ley del estado de Nueva York.

En rigor, se trató del segundo bono verde de Argentina durante el año 2017, luego de que la provincia de La Rioja hiciera lo propio, obteniendo de 200 millones de USD en febrero del mismo año. No obstante, La Rioja obtuvo una tasa del 9,75\% anual, mayor que la obtenida por Jujuy (Infobae, 2017).

La provincia previó que el financiamiento del bono se realice con los ingresos obtenidos por la venta de la energía que genere el parque solar de Cauchari (Dirección de Prensa del Gobierno de Jujuy, 2017). Por lo tanto, se trató de un bono que no cuenta con garantía de los fondos de coparticipación, habiéndose acordado que su pago se efectúe de forma íntegra con los fondos generados por el mismo proyecto. Esta estrategia de gestión trasnacional empleada por Jujuy, es congruente con el marco jurídico argentino que reconoce a las provincias la capacidad de realizar acciones en el escenario internacional siempre que no afecten el crédito público del Estado (Constitución Nacional Argentina, Artículo 124).

En este sentido, para el gobernador jujeño Gerardo Morales, "las características del bono, ligado al parque solar y la estrategia de desarrollo de la provincia en materia de energías renovables y litio, fue la clave del éxito" (Ámbito Financiero, 2017).

No obstante, el inicio del funcionamiento de los parques previsto para mayo de 2018, fue postergado en varias oportunidades debido a demoras de la licitación de las obras de línea de alta tensión, a cargo del Estado nacional (Jujuy al Momento, 2018). Este retraso supuso un importante problema: los plazos para que la provincia comience a pagar las deudas contraídas empezaron a correr antes de que la planta esté en funcionamiento, y, por lo tanto, la Jujuy debió afrontar estas obligaciones cuando no contaba con un producto para vender. Con las obras acabadas, Cauchari comenzó a producir energía para la red nacional en julio de 2020, dos años después de la fecha prevista.

La gestión subestatal del parque solar puso en relieve distintas instancias de cooperación y tensión entre Jujuy, el Estado y los actores chinos. La sinergia inicial entre el gobierno provincial y el nacional a comienzos del proyecto, muestra una gestión coherente y colaborativa que visibilizó una coherencia de intereses ante los actores chinos, que se tradujo en la concreción de las inversiones posteriores. No obstante, también se observaron acciones de coordinación ineficientes entre la provincia y los organismos nacionales en el momento de la construcción del sistema de interconexión de la planta con la red nacional de energía, ocasionando el retraso de la obra y las consecuencias derivadas (Juste, 2020).

\section{CONCLUSIONES}

A partir de la década de 1990, los Estados latinoamericanos iniciaron un periodo de reformas en sus marcos legales, regulatorios y por modernizar sus 
instituciones para atraer las inversiones extranjeras. Si bien estos cambios introducidos han permitido la llegada de inversiones, se ha criticado fuertemente el impacto de la IED y los proyectos que financian sobre los recursos naturales y el ambiente. Además, la explotación de los recursos naturales, en la mayoría de los casos, no ha logrado integrar otras actividades productivas, ni el desarrollo de cadenas de valor que impacten de manera positiva en las economías regionales y consecuentemente, generen desarrollo.

A partir del 2005, el "boom del litio" en el escenario internacional significó una oportunidad para una reinserción de Argentina en materia minera. Diez años más tarde, en 2015, el interés se centró en desarrollar la energía solar, en búsqueda de una transformación de la matriz energética y productiva de la provincia. Ambos hechos abrieron la participación de provincias periféricas como Jujuy, que se involucró de manera activa en la gestión de sus recursos, articulando con actores nacionales e internacionales en pos del desarrollo subestatal.

Este cambio hacia el desarrollo de energías "verdes" se vio favorecido por factores globales como el crecimiento económico de los países asiáticos que benefició a las regiones de recursos medioambientales atractivos, como Jujuy. El cambio de gestión gubernamental a nivel estatal y subestatal, aportó una visión de apertura comercial que, junto a la eliminación de retenciones a la producción minera, y a las políticas de vinculación con institutos científicos, propició la articulación entre el Estado provincial, el sector privado, y la ciencia.

La clave para el éxito de los proyectos que se anclan en Jujuy, parece estar en la coordinación de las gestiones entre los niveles estatal y subestatal, de manera de poder canalizar de mejor manera los beneficios de las inversiones. Esto se observa en la construcción de la planta solar de Cauchari, donde la demora de las obras a cargo del Estado nacional, retrasa el inicio del funcionamiento de la planta y, por consiguiente, perjudica la obtención de los fondos para responder a las obligaciones financieras contraídas por la provincia para su construcción. En el caso de los proyectos de litio, la fortaleza de la vinculación Estado-unidad subestatal y actores privados, radica en el diseño de estrategias que incorporen la necesidad de desarrollar valor agregado a la producción exportable.

Asimismo, si se piensa en los beneficios para el desarrollo local que posibilitan la producción de energía solar y litio, se visualizan tres campos. Los beneficios inmediatos tienen que ver con la generación de empleo a través de la creación de empresas proveedoras de servicios, y el retorno de ganancias a través de las regalías mineras y de la "renta minera" que obtenga la empresa estatal JEMSE en el caso del litio. En un futuro, a ello hay que sumar el hecho de que Jujuy empezará a comercializar la energía obtenida en Cauchari a CAMESA, la empresa que regula el interconectado y distribución de energía eléctrica del país.

Por otro lado, las inversiones propulsan el sector productivo jujeño en dos sentidos: 1) sumando capital y aumentando su capacidad de producción; 2) la transferencia de técnicas y tecnologías que permiten mejorar la productividad. Además, se pueden mencionar temas relacionados al desarrollo del mercado interno, la inserción en mercados internacionales, la especialización de mano de obra y el desarrollo de circuitos de proveedores locales, etc.

Como se mencionó antes, lo interesante de la producción de litio y de energía solar en Jujuy, consiste en la articulación de diferentes actores del ámbito nacional 
y provincial a fin de descentralizar y reproducir los beneficios de la inversión. Tanto la experiencia del litio como la de Cauchari, permiten observar el surgimiento de nuevos actores como la Organización Federal de los Estados Mineros (OFEMI); la intervención de otros como la Universidad Nacional, JEMSE, entre otros; la participación de empresas locales y comunidades de pueblos originarios. Estos actores trabajaron en forma conjunta los proyectos de exploración y explotación en la búsqueda de oportunidades de generación de servicios, empleo y conservación del medioambiente. En este sentido, se observa que para que la gestión transnacional de las unidades subestatales propenda al desarrollo, se requiere una articulación eficiente entre actores locales, nacionales y (públicos y privados), que posibiliten maximizar los beneficios de la IED y derramarlos hacia la economía regional y nacional.

No obstante, resta averiguar si esta articulación podrá transformarse en un futuro en el desarrollo de conocimiento y su posterior aplicación tecnológica a nivel local, para la cual se encuentran actualmente avocados el gobierno provincial, la universidad y el sector privado. La comunicación y la labor conjunta de estas tres partes permitirán generar los canales para el mejor aprovechamiento de la IED y de los recursos naturales del suelo jujeño.

La realidad actual evidencia que las unidades subestatales han iniciado un proceso de despliegue de acciones políticas en diferentes esferas con el objetivo de reposicionar sus intereses y perseguir sus objetivos de desarrollo local, en virtud de las oportunidades y desafíos del contexto. Estas gestiones se desarrollan en dos planos de acciones: estatal e internacional. En el primer plano, la provincia coordina con el Estado nacional acciones que no solo vayan acorde a la política exterior, sino que puedan reforzar los intereses subestatal en el escenario internacional. En el segundo plano, Jujuy inició vinculaciones con actores internacionales, privados y públicos, con el fin de atraer inversiones y cooperación técnica que le permita adquirir nuevas habilidades y desarrollar sus recursos. Es decir, de manera complementaria a las inversiones que provinieron, tradicionalmente, del Estado nacional.

Una de las consecuencias observables como producto de este fenómeno a nivel subestatal, es un proceso de cambio en la matriz productiva desde una economía regional primaria orientada a la producción de tabaco, azúcar y minería hacia una economía de producción energética "verde". La segunda consecuencia, es un reposicionamiento de los intereses de desarrollo de la provincia dentro de la agenda del Estado federal. Esto último se debe a una congruencia entre los intereses de la política exterior del Estado y las acciones de gestión transnacional de Jujuy que, como resultado, han producido una agenda de trabajo y coordinación entre ambos niveles de gobierno.

Estas acciones no solo beneficia en términos de crecimiento y desarrollo local para una economía periférica como la jujeña, sino además, manifiesta a nivel internacional, una congruencia entre la gestión subestatal transnacional y la política exterior del Estado. En este último sentido, la gestión transnacional subestatal profundiza la política estatal de la administración actual, instrumentándola en acciones concretas; $\mathrm{y}$, viceversa, la política exterior estatal contribuye a fortalecer y dar sustento a los intereses de desarrollo local provinciales. 


\section{BIBLIOGRAFÍA}

Alburquerque, F. (2001). "Desarrollo Económico Local y Descentralización en América Latina". Revista de la CEPAL, Abril, No 82: 157-171.

Boisier, S. (2001). "Desarrollo (local): ¿de qué estamos hablando?". En: Transformaciones globales, instituciones y políticas de desarrollo local (pp. 48-74). Vázquez Barquero, A. y Madoery, O., editores. Rosario: Editorial Homo Sapiens.

Calvento, M. (2014). "La inserción internacional de los actores subnacionales: análisis de un proceso contemporáneo". Revista Interaçoes, Campo Grande, V.15, $\mathrm{N}^{\circ}$ 2: 301-313.

Cardoso, F. y Faletto, E. (1977). Dependencia y Desarrollo en América Latina. Buenos Aires: Siglo XXI.

Castello, A. y Kloster, M. (2015). "Industrialización del litio y agregado de valor local: informe tecno-productivo". En publicación del Centro interdisciplinario de Estudios en Ciencia, Tecnología e Innovación. Buenos Aires: Ministerio de Ciencia, Tecnología e Innovación Productiva.

Colacrai, M. y Zubelzú, G. (1994). "Las provincias y sus relaciones externas ¿federalización de la política exterior o protagonismo provincial en las relaciones internacionales?". Revista Cuadernos de Política Exterior Argentina del Centro de Estudios en Relaciones Internacionales de Rosario, Serie Documentos de Trabajo $\mathrm{N}^{\circ} 6:$ : 1-47.

Comisión Nacional de Litio de Chile, (2015). Informe: "Litio: una fuente de energía, una oportunidad para Chile", Ministerio de Minería de Chile, Santiago de Chile.

Del Arenal, C. (2009). "Mundialización, creciente interdependencia y globalización en las relaciones internacionales". Cursos de Derecho Internacional y Relaciones Internacionales. Vitoria-Gasteiz, Universidad del País Vasco, Bilbao.

Dos Santos, T. (2011). Imperialismo y Dependencia. Caracas: Colección Claves Políticas de América, $\mathrm{N}^{\circ} 5$.

Duchaceck, I. (1986). "Las dimensiones territoriales de las políticas: dentro, entre y a través de las Naciones". Westview Press Boulder-Colorado.

Fornillo, B. (2015) "Del salar a la batería: Política, ciencia e industria del litio en la Argentina". En: Geopolítica del Litio: industria, ciencia y energía en Argentina (pp. 57-90). Bruno Fornillo, coordinador. CLACSO, Buenos Aires.

García Segura, C. (1996). "La actividad exterior de las entidades políticas subestatales”, Revista de Estudios Políticos Nueva Época, Madrid, V. Eneromarzo, $\mathrm{N}^{\circ}$ 91: 234-264.

Juste, S. (2017) "La condición de doble periferia en unidades subestatales". Revista Interações, Campo Grande, $\mathrm{N}^{\circ}$ 18(4): 169-184.

(2020) "La agenda subestatal de las relaciones argentino-chinas. La gestión transnacional de las provincias argentinas durante la administración de Macri”. Revista Intellector, Rio de Janeiro, Ano XVI, Volume XVI, No 32: 2840.

Keating, M. (1993). "Regionalismo, autonomía y relaciones internacionales", University of Western Ontario, Working Paper, $\mathrm{N}^{\circ} 66$. 
Keohane, R. y Nye, J. (1989). Poder e interdependencia. La política mundial en transición. Buenos Aires: Grupo Editor Latinoamericano.

Ministerio de Economía y Finanzas Públicas de Argentina (MECON) (2016). Ficha Provincial: Jujuy, Octubre de 2016, Secretaría de Política Económica y Planificación del Desarrollo. Disponible en: $<$ http://www.mecon.gov.ar/peconomica/dnper/fichas_provinciales/Jujuy.p df $>$.

Oviedo, E. (2017). "Alternancia política y capitales chinos en Argentina". En: Inversiones de China, Corea y Japón en Argentina: análisis general y estudio de casos (pp.12-35). Eduardo Oviedo, coordinador. Rosario: UNR Editora.

Roseneau, J. (1997). "Demasiadas cosas a la vez. La teoría de la complejidad y de los asuntos mundiales". Revista Nueva Sociedad, Buenos Aires, N $^{\circ}$ 148: 70-83.

Rusell, R. (2010). "El Estado - Nación y los actores gubernamentales no centrales: una relación complementaria". La Política Internacional Subnacional en América Latina (pp. 83-106). Luis Maira, editor, Buenos Aires: El Zorzal.

Sarquís, D. (2009). "Paradiplomacia: fundamentos teóricos". Regionalización y Paradiplomacia: la política internacional de las regiones (pp. 29-66). Zidane Zeraoui, coordinador. Ciudad de México: Editorial Montiel \& Soriano.

Prebisch, R. (2008). Hacia una teoría en transformación. Revista de la CEPAL, Santiago de Chile, $\mathrm{N}^{\circ} 6$.

Slipak, Ariel, (2015). "La extracción del litio en la Argentina y el debate sobre la "riqueza natural". En: Geopolítica del Litio: industria, ciencia y energía en Argentina (pp. 91-122). Fruno Fornillo, coordinador. Buenos Aires: CLACSO.

Schujovitzky, L. (2017). "Capitales chinos en Argentina: naturaleza y clasificación en base a casos de estudio". En: Inversiones de China, Corea y Japón en Argentina: análisis general y estudio de casos (pp. 36-52). Eduardo Oviedo, coordinador. Rosario: UNR Editora.

Wallerstein, I. (1979). El moderno sistema mundial I: La agricultura capitalista y los Orígenes de la economía-mundo europea en el siglo XVI. Madrid: Siglo XXI, $2 \mathrm{a}$ ed.

Zeraoui, Z. (2009). Regionalización y Paradiplomacia: la política internacional de las regiones. Ciudad de México: Editorial Montiel \& Soriano.

Zubelzú, G. (2006). "La acción internacional de las provincias argentinas. Reflexiones generales y análisis de algunos perfiles de gestión”. Revista Estudios del Centro de Estudios Avanzados (CEA) de la Universidad Nacional de Córdoba, ciudad de Córdoba, $\mathrm{N}^{\circ}$ 18: 147-160.

\section{RECURSOS DE LA WEB}

Diario Ámbito (2017, 14 de septiembre ), "Jujuy colocó un "bono verde" por u \$s210 M (8,62\%)", Buenos Aires, Argentina. Disponible en: http://www.ambito.com/897128-jujuy-coloco-un-bono-verde-por-us210-m862

Dirección de Prensa del Gobierno de Jujuy (2016, 30 de septiembre), "Histórico: Jujuy ganó la licitación para generar 300 Mw de energía solar”, San Salvador de Jujuy, Argentina. Disponible en: http://prensa.jujuy.gob.ar/2016/o9/30/historico-jujuy-gano-la-licitacionpara-generar-300-mw-de-energia-solar/ 
Dirección de Prensa del Gobierno de Jujuy (2017, 8 de septiembre), "Bono Verde para Cauchari”, San Salvador de Jujuy, Argentina. Disponible en: http://prensa.jujuy.gob.ar/2017/o9/o8/bono-verde-para-cauchari/

Dirección de Prensa del Gobierno de Jujuy (2019, 11 de septiembre), "Lanzaron el proyecto de expansión de Sales de Jujuy”, San Salvador de Jujuy, Argentina. Disponible en: http://prensa.jujuy.gob.ar/2019/o9/11/lanzaron-el-proyectode-expansion-de-sales-de-jujuy/

EconoJournal (2017, s/f) "Comienza en agosto un proyecto de litio por US\$ 500 millones", Buenos Aires, Argentina. Disponible en: http://econojournal.com.ar/2017/05/comienza-en-agosto-un-proyecto-delitio-por-us-500-millones/.

Ecopost (2016, 12 de julio), "La credibilidad de los bonos verdes y su enorme potencial”. Disponible en: https://www.ecopost.info/la-credibilidad-de-losbonos-verdes-y-su-enorme-potencial/.

El Tribuno de Jujuy (2017, 16 de mayo), "Jujuy recibirá un crédito millonario para construir un parque solar”, San Salvador de Jujuy, Argentina. Disponible en: http://www.eltribuno.info/salta/nota/2017-5-16-17-18-0-jujuy-recibira-uncredito-millonario-para-construir-un-parque-solar

El Tribuno de Jujuy (2017, 24 de mayo), "Jujuy avanza hacia la instalación del parque solar", San Salvador de Jujuy, Argentina. Disponible en: http://www.eltribuno.info/jujuy/nota/2017-5-23-22-22-0-jujuy-avanzahacia-la-instalacion-del-parque-solar

El Tribuno de Jujuy (2017, 13 de junio), "Anuncian la fabricación de baterías de litio en Jujuy", San Salvador de Jujuy, Argentina. Disponible en: http://www.eltribuno.info/jujuy/nota/2017-6-12-22-8-o-anuncian-lafabricacion-de-baterias-de-litio-en-jujuy

Forbes Argentina (2017, 12 de agosto), "En búsqueda de inversiones chinas”, Buenos Aires, Argentina. Disponible en: http://www.forbesargentina.com/inversiones-chinas-plan-quinquenal/

Infobae (2017, 17 de febrero), "Bonos verdes: La Rioja colocó USD 200 millones en el exterior". Disponible en: https://www.infobae.com/economia/finanzas-ynegocios/2017/02/17/bonos-verdes-la-rioja-coloco-usd-200-millones-en-elexterior/.

Infobae (2017, 15 de agosto), "En China, Macri prometió que Argentina "duplicará la producción de alimentos" en los próximos años", Buenos Aires, Argentina. Disponible en: http://www.infobae.com/politica/2017/05/15/mauriciomacri-participo-de-un-foro-con-lideres-mundiales-en-china/

Jujuy Al Momento (2018, 22 de febrero). "La planta solar funcionará recién en 2019", San Salvador de Jujuy, Argentina. Disponible en: http://www.jujuyalmomento.com/post/81447/la-planta-solar-funcionararecien-en-2019.html

Jujuy Online (2017, 24 de agosto), "Litio: Inauguran Centro de Desarrollo Tecnológico", San Salvador de Jujuy, Argentina. Disponible en: http://www.jujuyonlinenoticias.com.ar/jujuy/2017/8/24/litio-inauguraroncentro-desarrollo-tecnologico-44601.html

Panorama Minero (2020, 30 de enero), "Cauchari-Olaroz: actualización del proyecto a enero de 2020", San Salvador de Jujuy, Argentina. Disponible en: 
https://panorama-minero.com/noticias/cauchari-olaroz-actualizacion-delproyecto-a-enero-de-2020/

Sales de Jujuy (s/f), Información General sobre el proyecto Olaroz. Disponible en: http://salesdejujuy.com/espanol/proyectos.

Télam (2017, 17 de agosto), "La lista de acuerdos firmados entre Argentina y China", Buenos Aires, Argentina. Disponible en: http://www.telam.com.ar/notas/201705/189339-acuerdos-argentinachina.html

Télam (2017, 24 de mayo), "Prevén instalar en Jujuy el segundo parque solar más grande del mundo", Buenos Aires, Argentina. Disponible en: http://www.telam.com.ar/notas/201705/190148-preven-instalar-en-jujuyel-segundo-parque-solar-mas-grande-del-mundo.html

Télam (2015, 25 de abril), "Esta semana se concreta la primera exportación de litio jujeño a Japón”, Buenos Aires, Argentina. Disponible en: http://www.telam.com.ar/notas/201504/102949-exportacion-litio-jujuyjapon.html 